\title{
Superstructure Reaction Network Design for the Synthesis of Biobased Sustainable Nitrogen-Containing Polymers
}

Thomas R. Savage ${ }^{1}$, Dongda Zhang, ${ }^{1, *}$

1: Centre for Process Integration, University of Manchester, Sackville Street, Manchester, M1 3AL, UK.

*: corresponding author, email: dongda.zhang@manchester.ac.uk, tel: 44 (0) 1613065153.

Table S1: The applications and potential use of all the 12 investigated nitrogen-containing polymers and the 3 nitrogen-free polymers included in the superstructure network.

Table S2: Summary of the reactions in the superstructure reaction network.

Table S3: Price of the raw material and products. 
Table S1: The applications and potential use of all the 12 investigated nitrogen-containing polymers and the 3 nitrogen-free polymers.

\begin{tabular}{|c|c|c|}
\hline Polymer & Level of production & Industrial Application \\
\hline Polyacrylnitrile (PAN) & Industrial Scale & $\begin{array}{l}\text { As a precursor for carbon } \\
\text { fibre manufacture. }\end{array}$ \\
\hline Polyacrylamide (PAM) & Industrial Scale & $\begin{array}{l}\text { Used in water treatment, } \\
\text { paper making and screen } \\
\text { printing. }\end{array}$ \\
\hline Nitrile rubber 1 (NBR1) & Industrial Scale & \multirow{2}{*}{$\begin{array}{l}\text { Uses as non-latex gloves and } \\
\text { many industrial components. }\end{array}$} \\
\hline Nitrile rubber 2 (NBR2) & Industrial Scale & \\
\hline Nylon 6,6 & Industrial Scale & $\begin{array}{l}\text { Large market with multiple } \\
\text { industrial and residential uses } \\
\text { due to its robustness. }\end{array}$ \\
\hline Nylon 6 & Industrial Scale & $\begin{array}{l}\text { Used for its high tensile } \\
\text { fibres and thermal resistance } \\
\text { has multiple industrial and } \\
\text { residential uses. }\end{array}$ \\
\hline Nylon 1,6 & Lab scale & $\begin{array}{l}\text { Currently, lab scale yet there } \\
\text { promises to be many uses. }\end{array}$ \\
\hline Polyurethane 1 (PEUR1) & Industrial Scale & \multirow{3}{*}{$\begin{array}{l}\text { Rigid foam used for multiple } \\
\text { industrial applications with } \\
\text { thermodynamic properties. }\end{array}$} \\
\hline Polyurethane 2 (PEUR2) & Industrial Scale & \\
\hline Polyurethane 3 (PEUR3) & Industrial Scale & \\
\hline Urea-formaldehyde (UF) & Industrial Scale & Fertiliser and adhesive use. \\
\hline Polyaniline (PANI) & Lab scale & $\begin{array}{l}\text { Conductive polymer, further } \\
\text { study required for uses } \\
\text { within electronics } \\
\text { investigated. }\end{array}$ \\
\hline Polypropylene (PP) & Industrial Scale & $\begin{array}{l}\text { Used in packaging and } \\
\text { labelling }\end{array}$ \\
\hline Polyethylene (PE) & Industrial Scale & Mainly used in packaging \\
\hline Polypropylene carbonate (PPC) & Industrial Scale & $\begin{array}{l}\text { Used as an additive in resins } \\
\text { and ceramics }\end{array}$ \\
\hline
\end{tabular}


Table S2: Summary of the reactions in the superstructure reaction network.

\begin{tabular}{|c|c|c|c|c|c|}
\hline & Reaction & $\mathbf{S}$ & $\mathbf{T}$ & $\Delta \mathbf{H}$ & Ref \\
\hline R1 & $\mathrm{CH}_{1.68} \mathrm{O}_{0.54}+0.23 \mathrm{O}_{2} \rightarrow \mathrm{CO}+0.84 \mathrm{H}_{2}$ & 100.0 & 973 & -372.1 & {$[1]$} \\
\hline R2 & $\mathrm{CH}_{4}+\mathrm{H}_{2} \mathrm{O} \rightarrow \mathrm{CO}+3 \mathrm{H}_{2}$ & 91.7 & 1200 & 250.3 & {$[2]$} \\
\hline $\mathrm{R} 3$ & $2 \mathrm{CO}+2 \mathrm{H}_{2} \rightarrow \mathrm{CH}_{4}+\mathrm{CO}_{2}$ & 80.0 & 500 & -318.7 & [3] \\
\hline R4 & $2 \mathrm{CO}+0.5 \mathrm{O}_{2}+4 \mathrm{H}_{2} \rightarrow \mathrm{C}_{2} \mathrm{H}_{6} \mathrm{O}_{2}+\mathrm{H}_{2} \mathrm{O}$ & 94.0 & 473 & -330.4 & [4] \\
\hline R5 & $\mathrm{C}_{7} \mathrm{H}_{8}+\mathrm{H}_{2} \rightarrow \mathrm{C}_{6} \mathrm{H}_{6}+\mathrm{CH}_{4}$ & 90 & 890 & -127.1 & [40] \\
\hline R6 & $2 \mathrm{CH}_{4} \rightarrow \mathrm{C}_{2} \mathrm{H}_{2}+3 \mathrm{H}_{2}$ & 90.0 & 1900 & 376.5 & [5] \\
\hline R7 & $\mathrm{CO}+2 \mathrm{H}_{2} \rightarrow \mathrm{CH}_{4} \mathrm{O}$ & 98.9 & 443 & -127.9 & {$[6]$} \\
\hline R8 & $2 \mathrm{CH}_{4} \mathrm{O} \rightarrow \mathrm{C}_{2} \mathrm{H}_{6} \mathrm{O}+\mathrm{H}_{2} \mathrm{O}$ & 87.5 & 573 & -64.43 & [7] \\
\hline R9 & $\mathrm{CH}_{4} \mathrm{O}+0.5 \mathrm{O}_{2} \rightarrow \mathrm{CH}_{2} \mathrm{O}+\mathrm{H}_{2} \mathrm{O}$ & 95.0 & 548 & -156.0 & [8] \\
\hline $\mathrm{R} 10$ & $\mathrm{CH}_{4} \mathrm{O}+\mathrm{CO}+2 \mathrm{H}_{2} \rightarrow \mathrm{C}_{2} \mathrm{H}_{6} \mathrm{O}+\mathrm{H}_{2} \mathrm{O}$ & 74.4 & 468 & -215.1 & [9] \\
\hline R11 & $\mathrm{CH}_{4}+\mathrm{CO}_{2} \rightarrow 2 \mathrm{CO}+2 \mathrm{H}_{2}$ & 100 & 1200 & 247.2 & [2] \\
\hline R12 & $\mathrm{C}_{2} \mathrm{H}_{6} \mathrm{O}+\mathrm{CH}_{4} \mathrm{O} \rightarrow \mathrm{C}_{3} \mathrm{H}_{6}+2 \mathrm{H}_{2} \mathrm{O}$ & 45.4 & 723 & -35.19 & [10] \\
\hline R13 & $\mathrm{C}_{3} \mathrm{H}_{6}+\mathrm{H}_{2}+\mathrm{O}_{2} \rightarrow \mathrm{C}_{3} \mathrm{H}_{6} \mathrm{O}+\mathrm{H}_{2} \mathrm{O}$ & 96.0 & 338 & -429.0 & [11] \\
\hline R14 & $\mathrm{C}_{6} \mathrm{H}_{6}+2 \mathrm{H}_{2} \rightarrow \mathrm{C}_{6} \mathrm{H}_{10}$ & 62.3 & 433 & -86.90 & [12] \\
\hline R15 & $3 \mathrm{C}_{2} \mathrm{H}_{2} \rightarrow \mathrm{C}_{6} \mathrm{H}_{6}$ & -- & 520 & -631.9 & [12] \\
\hline R16 & $2 \mathrm{C}_{2} \mathrm{H}_{6} \mathrm{O} \rightarrow \mathrm{C}_{4} \mathrm{H}_{6}+2 \mathrm{H}_{2} \mathrm{O}$ & 69.0 & 683 & 92.80 & [13] \\
\hline R17 & $\mathrm{C}_{2} \mathrm{H}_{2}+2 \mathrm{CH}_{2} \mathrm{O} \rightarrow \mathrm{C}_{4} \mathrm{H}_{6} \mathrm{O}_{2}$ & 90.0 & 373 & -100.0 & [14] \\
\hline R18 & $\mathrm{C}_{3} \mathrm{H}_{6} \mathrm{O}+\mathrm{H}_{2} \mathrm{O} \rightarrow \mathrm{C}_{3} \mathrm{H}_{8} \mathrm{O}_{2}$ & 93.0 & 473 & -67.70 & [15] \\
\hline R19 & $\mathrm{C}_{3} \mathrm{H}_{6} \mathrm{O}+\mathrm{CO}_{2} \rightarrow$ PPC & 99.9 & - & -68.2 & [16] \\
\hline R20 & $\mathrm{C}_{4} \mathrm{H}_{6} \mathrm{O}_{2}+2 \mathrm{H}_{2} \rightarrow \mathrm{C}_{4} \mathrm{H}_{10} \mathrm{O}_{2}$ & 95.0 & 453 & -251.0 & [14] \\
\hline $\mathrm{R} 21$ & $\mathrm{C}_{2} \mathrm{H}_{6} \mathrm{O} \rightarrow \mathrm{C}_{2} \mathrm{H}_{4}+\mathrm{H}_{2} \mathrm{O}$ & 99.7 & 513 & 44.3 & [17] \\
\hline R22 & $\mathrm{CO}+\mathrm{H}_{2} \mathrm{O} \rightarrow \mathrm{CO}_{2}+\mathrm{H}_{2}$ & 99.9 & 673 & -41.2 & [18] \\
\hline
\end{tabular}




\begin{tabular}{|c|c|c|c|c|c|}
\hline $\mathrm{R} 23$ & $\mathrm{C}_{2} \mathrm{H}_{4} \rightarrow \mathbf{P E}$ & 99.9 & - & -85.8 & [19] \\
\hline $\mathrm{R} 24$ & $\mathrm{C}_{3} \mathrm{H}_{6} \rightarrow \mathbf{P P}$ & 99.9 & - & -85.8 & [20] \\
\hline $\mathrm{R} 25$ & $\begin{array}{l}\mathrm{CH}_{1.589} \mathrm{O}_{0.402} \mathrm{~N}_{0.243}+0.299 \mathrm{O}_{2} \rightarrow \mathrm{CO}+0.43 \mathrm{H}_{2}+ \\
0.243 \mathrm{NH}_{3}\end{array}$ & 100 & 973 & -372.1 & [1] \\
\hline R26 & $\begin{array}{l}\mathrm{CH}_{1.589} \mathrm{O}_{0.402} \mathrm{~N}_{0.243} \rightarrow 0.243 \mathrm{NH}_{3}+0.048 \mathrm{C}_{6} \mathrm{H}_{6}+ \\
0.04 \mathrm{C}_{7} \mathrm{H}_{8}+0.024 \mathrm{C}_{8} \mathrm{H}_{10}+0.08 \mathrm{CO}+0.16 \mathrm{CO}_{2}\end{array}$ & 60 & 1173 & -372.1 & [21] \\
\hline R27 & $\mathrm{C}_{6} \mathrm{H}_{10}+2 \mathrm{H}_{2} \mathrm{O}_{2} \rightarrow \mathrm{C}_{6} \mathrm{H}_{10} \mathrm{O}_{4}+2 \mathrm{H}_{2}$ & 38.9 & 367 & 580.2 & {$[22]$} \\
\hline $\mathrm{R} 28$ & $2 \mathrm{C}_{3} \mathrm{H}_{6}+2 \mathrm{NH}_{3}+3 \mathrm{O}_{2} \rightarrow 2 \mathrm{C}_{3} \mathrm{H}_{3} \mathrm{~N}+6 \mathrm{H}_{2} \mathrm{O}$ & 70 & 673 & -1063.7 & [23] \\
\hline R29 & $\mathrm{C}_{3} \mathrm{H}_{3} \mathrm{~N} \rightarrow \mathbf{P A N}$ & 99.9 & - & -85.8 & {$[24]$} \\
\hline R30 & $\mathrm{C}_{3} \mathrm{H}_{3} \mathrm{~N}+\mathrm{H}_{2} \mathrm{O} \rightarrow \mathrm{C}_{3} \mathrm{H}_{5} \mathrm{NO}$ & 100 & 363 & -344.5 & {$[25]$} \\
\hline R31 & $\mathrm{C}_{3} \mathrm{H}_{5} \mathrm{NO} \rightarrow$ PAM & 99.9 & - & -85.8 & {$[26]$} \\
\hline $\mathrm{R} 32$ & $\mathrm{C}_{6} \mathrm{H}_{6}+3 \mathrm{H}_{2} \rightarrow \mathrm{C}_{6} \mathrm{H}_{12}$ & 100 & 403 & -206.3 & {$[27]$} \\
\hline $\mathrm{R} 33$ & $\mathrm{C}_{6} \mathrm{H}_{12}+\mathrm{O}_{2} \rightarrow \mathrm{C}_{6} \mathrm{H}_{10} \mathrm{O}+\mathrm{H}_{2} \mathrm{O}$ & 85 & 298 & -400.6 & {$[28]$} \\
\hline R34 & $\mathrm{C}_{6} \mathrm{H}_{10} \mathrm{O}+\mathrm{NH}_{3}+\mathrm{H}_{2} \mathrm{O}_{2} \rightarrow \mathrm{C}_{6} \mathrm{H}_{11} \mathrm{NO}+2 \mathrm{H}_{2} \mathrm{O}$ & 96.5 & 353 & -402.9 & [29] \\
\hline R35 & $2 \mathrm{C}_{3} \mathrm{H}_{3} \mathrm{~N}+\mathrm{H}_{2} \rightarrow \mathrm{C}_{6} \mathrm{H}_{8} \mathrm{~N}_{2}$ & 90 & 298 & -276.1 & {$[30]$} \\
\hline R36 & $\mathrm{C}_{6} \mathrm{H}_{8} \mathrm{~N}_{2}+4 \mathrm{H}_{2} \rightarrow \mathrm{C}_{6} \mathrm{H}_{16} \mathrm{~N}_{2}$ & 76 & 353 & -290.1 & {$[31]$} \\
\hline R37 & $\mathrm{C}_{6} \mathrm{H}_{11} \mathrm{NO} \rightarrow$ Nylon 6 & 99.9 & - & -85.8 & {$[32]$} \\
\hline R38 & $\mathrm{C}_{6} \mathrm{H}_{16} \mathrm{~N}_{2}+\mathrm{C}_{6} \mathrm{H}_{10} \mathrm{O}_{4} \rightarrow$ Nylon $\mathbf{6 , 6}+\mathrm{H}_{2} \mathrm{O}$ & 99.9 & - & -85.8 & {$[33]$} \\
\hline R39 & $\mathrm{C}_{6} \mathrm{H}_{8} \mathrm{~N}_{2}+\mathrm{CH}_{2} \mathrm{O}+\mathrm{H}_{2} \mathrm{O} \rightarrow$ Nylon $\mathbf{1 , 6}$ & 99.9 & - & -85.8 & {$[33]$} \\
\hline $\mathrm{R} 40$ & $\mathrm{C}_{3} \mathrm{H}_{3} \mathrm{~N}+(1,3) \mathrm{C}_{4} \mathrm{H}_{6} \rightarrow$ NBR1 & 99.9 & - & -85.8 & {$[34]$} \\
\hline R41 & $\mathrm{CO}_{2}+2 \mathrm{NH}_{3} \rightarrow\left(\mathrm{NH}_{2}\right)_{2} \mathrm{CO}+\mathrm{H}_{2} \mathrm{O}$ & 100 & 423 & -119 & {$[35]$} \\
\hline R42 & $\mathrm{C}_{6} \mathrm{H}_{12}+\mathrm{H}_{2} \mathrm{O}_{2} \rightarrow \mathrm{C}_{6} \mathrm{H}_{12} \mathrm{O}+\mathrm{H}_{2} \mathrm{O}+1 / 2 \mathrm{O}_{2}$ & 82.6 & 373 & -268.3 & {$[36]$} \\
\hline R43 & $\mathrm{C}_{6} \mathrm{H}_{12} \mathrm{O}+\mathrm{NH}_{3}+3 / 2 \mathrm{O}_{2} \rightarrow \mathrm{C}_{6} \mathrm{H}_{5} \mathrm{NH}_{2}+4 \mathrm{H}_{2} \mathrm{O}$ & 93 & 698 & -547.6 & {$[37]$} \\
\hline R44 & $\mathrm{C}_{6} \mathrm{H}_{5} \mathrm{NH}_{2}+\mathrm{CO}+\mathrm{C}_{2} \mathrm{H}_{5} \mathrm{OH}+1 / 2 \mathrm{O}_{2} \rightarrow \mathrm{C}_{9} \mathrm{H}_{11} \mathrm{NO}_{2}$ & 95 & 423 & -450.9 & {$[38]$} \\
\hline
\end{tabular}




\begin{tabular}{|l|l|l|l|l|l|}
\hline & $+\mathrm{H}_{2} \mathrm{O}$ & & & & \\
\hline $\mathrm{R} 45$ & $2 \mathrm{C}_{9} \mathrm{H}_{11} \mathrm{NO}_{2}+\mathrm{CH}_{2} \mathrm{O} \rightarrow \mathrm{C}_{15} \mathrm{H}_{10} \mathrm{~N}_{2} \mathrm{O}_{2}+2 \mathrm{C}_{2} \mathrm{H}_{5} \mathrm{OH}$ & 93 & 333 & -511.7 & {$[38]$} \\
\hline $\mathrm{R} 46$ & $\mathrm{C}_{15} \mathrm{H}_{10} \mathrm{~N}_{2} \mathrm{O}_{2}+\mathrm{C}_{2} \mathrm{H}_{6} \mathrm{O}_{2} \rightarrow$ PEUR1 & 99.9 & - & -85.8 & {$[39]$} \\
\hline $\mathrm{R} 47$ & $\mathrm{C}_{15} \mathrm{H}_{10} \mathrm{~N}_{2} \mathrm{O}_{2}+\mathrm{C}_{4} \mathrm{H}_{10} \mathrm{O}_{2} \rightarrow$ PEUR2 & 99.9 & - & -85.8 & {$[39]$} \\
\hline $\mathrm{R} 48$ & $\mathrm{C}_{15} \mathrm{H}_{10} \mathrm{~N}_{2} \mathrm{O}_{2}+\mathrm{C}_{3} \mathrm{H}_{8} \mathrm{O}_{2} \rightarrow$ PEUR3 & 99.9 & - & -85.8 & {$[39]$} \\
\hline $\mathrm{R} 49$ & $\left(\mathrm{NH}_{2}\right)_{2} \mathrm{CO}+\mathrm{CH}_{2} \mathrm{O} \rightarrow \mathbf{U F}$ & 99.9 & - & -85.8 & {$[39]$} \\
\hline $\mathrm{R} 50$ & $\mathrm{C}_{3} \mathrm{H}_{3} \mathrm{~N}+\mathrm{C}_{2} \mathrm{H}_{2} \rightarrow$ NRB2 & 99.9 & - & -85.8 & {$[33]$} \\
\hline R51 & $\mathrm{C}_{6} \mathrm{H}_{5} \mathrm{NH}_{2} \rightarrow$ PANI & 99.9 & - & -85.8 & {$[39]$} \\
\hline
\end{tabular}

1. S: selectivity; T: temperature $(\mathrm{K})$; Ref: reference; $\Delta \mathrm{H}$ : reaction enthalpy $(\mathrm{kJ} / \mathrm{kmol})$;

2. Conversion efficiencies of all current reactions are assumed to be $99.0 \%$, as in practice unconverted reactants will be recovered by separation units and then sent back to the reactor for further reaction. Selectivity of reaction in the absence of data is assumed to be $75 \%$ (empirical information);

3. The conversion efficiency and selectivity of polymerisation is assumed to be $99.0 \%$ and $99.9 \%$, correspondingly unless stated otherwise. 
Table S3: Price of the raw material and products. Price of biomass wastes comes from the previous study [41], price of other raw materials is obtained from the previous study [42], price of all the products come from the international manufacturer website Ailbaba.com.

\begin{tabular}{|l|l|l|l|}
\hline Chemical & Price $(\$ / \mathrm{kg})$ & Chemical & Price $(\$ / \mathrm{kg})$ \\
\hline $\mathrm{H}_{2} \mathrm{O}_{2}$ & 0.39 & $\mathrm{H}_{2}$ & 4.00 \\
\hline Biomass waste & 0.05 & $\mathrm{NH}_{3}$ & 2.00 \\
\hline PPC & 1.15 & $\mathrm{H}_{2} \mathrm{O}$ & 0.0005 \\
\hline PE & 0.91 & NBR1 & 2.24 \\
\hline PP & 1.73 & NBR2 & 2.24 \\
\hline PAN & 2.50 & PEUR1 & 2.45 \\
\hline PAM & 2.00 & PEUR2 & 2.73 \\
\hline Nylon 6 & 3.00 & PEUR3 & 2.59 \\
\hline Nylon 6,6 & 3.50 & UF & 0.85 \\
\hline Nylon 1,6 & 2.50 & PANI & 3.50 \\
\hline
\end{tabular}




\section{References:}

[1] Sugathapala A. (2013). Technologies for Converting Waste Agricultural Biomass to Energy. United Nations Environmental Programme, Osaka.

[2] Choudhary, V., Uphade, B. and Mamman, A. (1995). Large enhancement in methane-tosyngas conversion activity of supported Ni catalysts due to precoating of catalyst supports with $\mathrm{MgO}, \mathrm{CaO}$ or rare-earth oxide. Catalysis Letters, 32(3-4), pp.387-390.

[3] Hwang, S., Lee, J., Hong, U., Jung, J., Koh, D., Lim, H., Byun, C. and Song, I. (2012). Hydrogenation of carbon monoxide to methane over mesoporous nickel-M-alumina $(\mathrm{M}=\mathrm{Fe}$, $\mathrm{Ni}, \mathrm{Co}, \mathrm{Ce}$, and La) xerogel catalysts. Journal of Industrial and Engineering Chemistry, 18(1), pp.243-248.

[4] Luo, N., Ji, Y., Mao, Y. and Zhang, B. (2012). Syn-gas-based mono ethylene glycol synthesis in Pujing Chemical. Applied Petrochemical Research, 2(1-2), pp.23-26.

[5] Holmen, A., Olsvik, O. and Rokstad, O. (1995). Pyrolysis of natural gas: chemistry and process concepts. Fuel Processing Technology, 42(2-3), pp.249-267.

[6] Reubroycharoen, P., Yamagami, T., Vitidsant, T., Yoneyama, Y., Ito, M. and Tsubaki, N. (2003). Continuous Low-Temperature Methanol Synthesis from Syngas Using Alcohol Promoters. Energy \& Fuels, 17(4), pp.817-821.

[7] Yaripour, F., Baghaei, F., Schmidt, I. and Perregaard, J. (2005). Catalytic dehydration of methanol to dimethyl ether (DME) over solid-acid catalysts. Catalysis Communications, 6(2), pp.147-152.

[8] Dai, W., Liu, Q., Cao, Y. and Deng, J. (1998). Oxidative dehydrogenation of methanol to formaldehyde on electrolytic silver catalyst modified with iodides. Applied Catalysis A: General, 175(1-2), pp.83-88. 
[9] Francoisse, P. and Thyrion, F. (1983). Methanol to ethanol by homologation: kinetic approach. Industrial \& Engineering Chemistry Product Research and Development, 22(4), pp.542-548.

[10] Zhao, T., Takemoto, T. and Tsubaki, N. (2006). Direct synthesis of propylene and light olefins from dimethyl ether catalyzed by modified H-ZSM-5. Catalysis Communications, 7(9), pp.647-650.

[11] Zuwei, X. (2001). Reaction-Controlled Phase-Transfer Catalysis for Propylene Epoxidation to Propylene Oxide. Science, 292(5519), pp.1139-1141.

[12] Liu, Z., Xie, S., Liu, B. and Deng, J. (1999). Benzene-selective hydrogenation to cyclohexene over supported ruthenium boride catalysts prepared by a novel method. New Journal of Chemistry, 23(11), pp.1057-1057.

[13] Makshina, E., Janssens, W., Sels, B. and Jacobs, P. (2012). Catalytic study of the conversion of ethanol into 1,3-butadiene. Catalysis Today, 198(1), pp.338-344.

[14] LookChem. Manufacture of 1,4-butanediol. http://www.lookchem.com/Chempedia/C hemical\%ADTechnology/Organic\%ADChemical\% ADTechnology/7495.html.

[15] Propylene glycol Wikipedia. https://en.wikipedia.org/wiki/Propylene_glycol.

[16] von der Assen, N. and Bardow, A. (2014). Life cycle assessment of polyols for polyurethane production using $\mathrm{CO} 2$ as feedstock: insights from an industrial case study. Green Chem., 16(6), pp.3272-3280.

[17] Fan, D., Dai, D. and Wu, H. (2012). Ethylene Formation by Catalytic Dehydration of Ethanol with Industrial Considerations. Materials, 6(1), pp.101-115.

[18] Sharma, S. and Srivastava, A. (2003). Alternating Copolymers of Limonene with Methyl Methacrylate: Kinetics and Mechanism. Journal of Macromolecular Science, Part A, 40(6), pp.593-603. 
[19] Wang, C., Friedrich, S., Younkin, T., Li, R., Grubbs, R., Bansleben, D. and Day, M. (1998). Neutral Nickel(II)-Based Catalysts for Ethylene Polymerization. Organometallics, 17(15), pp.3149-3151.

[20] Li, G., Lamberti, M., Mazzeo, M., Pappalardo, D. and Pellecchia, C. (2013). Isospecific polymerization of propene by new indolyl-pyridylamido $\operatorname{Zr}(\mathrm{IV})$ catalysts. Journal of Molecular Catalysis A: Chemical, 370, pp.28-34.

[21] Wang, K. and Brown, R. (2013). Catalytic pyrolysis of microalgae for production of aromatics and ammonia. Green Chemistry, 15(3), pp.675.

[22] Deng Y, Ma Z, Wang K, Chen J, (1999). Clean synthesis of adipic acid by direct oxidation of cyclohexene with $\mathrm{H} 2 \mathrm{O} 2$ over peroxytungstate-organic complex catalysts. Green Chemistry December 1999. 275-276.

[23] Guo, C.J (1999). Science and Technology in Catalysis 1998. Tokyo: Proceedings of the Third Tokyo Conference on Advanced Catalytic Science and Technology, pp.347-350.

[24] Schaper, F., Foley, S. and Jordan, R. (2004). Acrylonitrile Polymerization by Cy3PCuMe and (Bipy)2FeEt2. Journal of the American Chemical Society, 126(7), pp.21142124.

[25] Kaushik, V. and Ravindranathan, M. (1989). ESCA study of Copper Catalysts Used for the Hydration of Acrylonitrile to Acrylamide. Applied Catalysis, 47(2), pp.343-349.

[26] Bera, P. and Saha, S. (1998). Redox polymerisation of acrylamide on aqueous montnorillonite surface: kinetics and mechanism of enhanced chain growth. Polymer, 39(67), pp.1461-1469.

[27] Vangelis, C., Bouriazos, A., Sotiriou, S., Samorski, M., Gutsche, B. and Papadogianakis, G. (2010). Catalytic conversions in green aqueous media: Highly efficient biphasic hydrogenation of benzene to cyclohexane catalyzed by $\mathrm{Rh} / \mathrm{TPPTS}$ complexes. Journal of Catalysis, 274(1), pp.2128. 
[28] Sarkar, B., Prajapati, P., Tiwari, R., Tiwari, R., Ghosh, S., Shubhra Acharyya, S., Pendem, C., Kumar Singha, R., Sivakumar Konathala, L., Kumar, J., Sasaki, T. and Bal, R. (2012). Room temperature selective oxidation of cyclohexane over Cu-nanoclusters supported on nanocrystalline Cr2O3. Green Chemistry, 14(9), p.2600.

[29] Zong, B., Sun, B., Cheng, S., Mu, X., Yang, K., Zhao, J., Zhang, X. and Wu, W. (2017). Green Production Technology of the Monomer of Nylon-6: Caprolactam. Engineering, 3(3), pp.379-384.

[30] Suwanvaipattana, P., Limtrakul, S., Vatanatham, T. and Ramachandran, P. (2017). Modeling of electro-organic synthesis to facilitate cleaner chemical manufacturing: Adiponitrile production. Journal of Cleaner Production, 142, pp.1296-1308.

[31] Alini, S., Bottino, A., Capannelli, G., Carbone, R., Comite, A. and Vitulli, G. (2003). The catalytic hydrogenation of adiponitrile to hexamethylenediamine over a rhodium/alumina catalyst in a three phase slurry reactor. Journal of Molecular Catalysis A: Chemical, 206(12), pp.363-370.

[32] Khodabakhshi, K., Gilbert, M., Fathi, S. and Dickens, P. (2013). Anionic polymerisation of caprolactam at the small-scale via DSC investigations. Journal of Thermal Analysis and Calorimetry, 115(1), pp.383-391.

[33] Magat, E., Chandler, L., Faris, B., Reith, J. and Salisbury, L. (1951). Acid-catalyzed Reactions of Nitriles. II. Polyamides from Formaldehyde and Dinitriles. Journal of the American Chemical Society, 73(3), pp.1031-1035.

[34] Cheng, T., Chen, J., Cai, A., Wang, J., Liu, H., Hu, Y., Bao, X. and Yuan, P. (2018). Synthesis of $\mathrm{Pd} / \mathrm{SiO} 2$ Catalysts in Various $\mathrm{HCl}$ Concentrations for Selective NBR Hydrogenation: Effects of $\mathrm{H}+$ and $\mathrm{Cl}-$ Concentrations and Electrostatic Interactions. $A C S$ Omega, 3(6), pp.6651-6659. 
[35] Barzagli, F., Mani, F. and Peruzzini, M. (2016). Carbon dioxide uptake as ammonia and amine carbamates and their efficient conversion into urea and 1,3-disubstituted ureas. Journal of $\mathrm{CO}_{2}$ Utilization, 13, pp.81-89.

[36] Yao, W., Chen, Y., Min, L., Fang, H., Yan, Z., Wang, H. and Wang, J. (2006). Liquid oxidation of cyclohexane to cyclohexanol over cerium-doped MCM-41. Journal of Molecular Catalysis A: Chemical, 246(1-2), pp.162-166.

[37] Manohar, B., Ganesh, I. and Reddy, B. (1998). Aniline synthesis from cyclohexanol and ammonia over mixed oxide catalysts. Journal of Molecular Catalysis A: Chemical, 129(1), pp.L5-L8.

[38] Chono, M., Fukuoka, S. and Kohno, M. (1983). A New Route to MDI without Phosgene. Journal of Cellular Plastics, 19(6), pp.385-390.

[39] Sonnenschein, Mark F. (2014). Polyurethanes: Science, Technology, Markets, and Trends. John Wiley \& Sons, Inc: Hoboken, NJ, 2014.

[40] Doumani, T. (1958). Dealkylation of Organic Compounds. Benzene from Toluene. Industrial \& Engineering Chemistry, 50(11), pp.1677-1680.

[41] Zhang, D., del Rio-Chanona, E. A., Shah, N. (2017). Screening Synthesis Pathways for Biomass-Derived Sustainable Polymer Production. ACS Sustain. Chem. Eng. 5(5), pp. 43884398.

[42] Ulonska K, Voll A, Marquardt W. (2016). Screening Pathways for the Production of Next Generation Biofuels. Energy \& Fuels.30(1), pp.445-456. 